\title{
Web based Secure Handout Management System for Professional Master's Students at Jahangirnagar University
}

\author{
Md. Atikur Rahman \\ Dept. of CSE \\ Gono Bishwabidyalay \\ Dhaka, Bangladesh
}

\author{
Md. Shahriare Satu \\ Dept. of CSE \\ Gono Bishwabidyalay \\ Dhaka, Bangladesh
}

\author{
Md. Rasel Mia \\ Dept. of CSE \\ Gono Bishwabidyalay \\ Dhaka, Bangladesh
}

\author{
K. M. Akkas Ali \\ I.I.T. \\ Jahangirnagar University \\ Dhaka, Bangladesh
}

\begin{abstract}
Handout management system provides a simple interface that is used to maintain class notes of the instructor in different courses. So, it provides an opportunity to upload handouts by the instructors, maintain them and students are downloaded their required notes from this system. So, it is useful for different educational institutes (schools, colleges and universities) to maintain records of class notes on the web. In this work, there are implemented handout management system of professional master's student at the Department of Computer Science and Engineering in Jahangirnagar University that observes some criteria about storage, upload and download time of handouts and shows a graph of different courses and student which indicates student interest in different courses using secure https protocol. Then, it is showed satisfactory outcomes which is helpful to measure useful criteria to create and manipulate secure handout management system on the web.
\end{abstract}

\section{Keywords}

Handout, Student, Instructor, Course

\section{INTRODUCTION}

Bangladesh is a developing country that contains many educational institutions which are organized by different processes like information, result, handout processing etc. Most of the educational institutions are still followed traditional process to do these kinds of task in this country. But, this is so much tedious and time consuming. Handout management is one of the most important task in the educational institutions where class materials are provided to the students by the course instructors. In this situation, web based handout management system is the best solution to manipulate this tasks. So, there are developed a web based secure handout management system for the professional master's students at the Department of Computer Science and Engineering in Jahangirnagar University. At first, educational staff are uploaded sufficient information about students, course instructors and courses of this program. Then, instructors are uploaded course materials(notes/files/documents) which courses are taken by him. On the other hand, assigned students of these particular course find out their course materials from his account. To make secure transaction between web application and database management system, https protocol is used in this application. Then, there are compared different upload and download times and correlate some courses with number of assigned students to find out student interest about different courses in this program.

In this paper, section II describes relevant works which are related to this system. Section III defines different requirements of this system. Then, Section IV describes about different working modules in this system. Section V describes the result and relevant discussion about this system. Section VI concludes by giving brief details of this system and indicates some future works about it.

\section{RELATED WORKS}

There are developed different types of management systems all over the world. But, there are described some relevant tasks which is related to this work. Bimal Aklesh Kumar [1] develop a thin client web-based campus information system for Fiji National University that provides a single point of access to all secure administrative systems at higher education sector. S.R.Bharamagoudar et.al [2] describe a web based student information management system which utilizes authentication services for the users and they can create, update and watch their information in this system. Bahubali Akiwate et al. [3] develop a web based student information management system that displays required information for each user using MEAN Stack. Rui Duan et.al [4] implement a web-based management information system using contingency theory and manage graduate \& other academic education from the interrelation and dynamic activity more efficiently in the system. Mecca, Giansalvatore, et al. [5] develop araneus web-based management system which represents a proposal of data-repository to manage web data. Huang et al. [6] implement a secure web-enabled workflow management framework for specification and enforcement of complex security policies within a workflow. Memon, Adeel Akbar, et al. [7] develop a new web based student annual review information system (SARIS) with a prediction method for successful students by using different data mining methods. Yevale, Prafulla S. et al. [8] develop a web based college information monitoring system using Java (DigiCampus). Izang, Aaron A., et al. [9] implement a web-based project management system that monitors project progress, allocates tasks, creates milestones and provides 


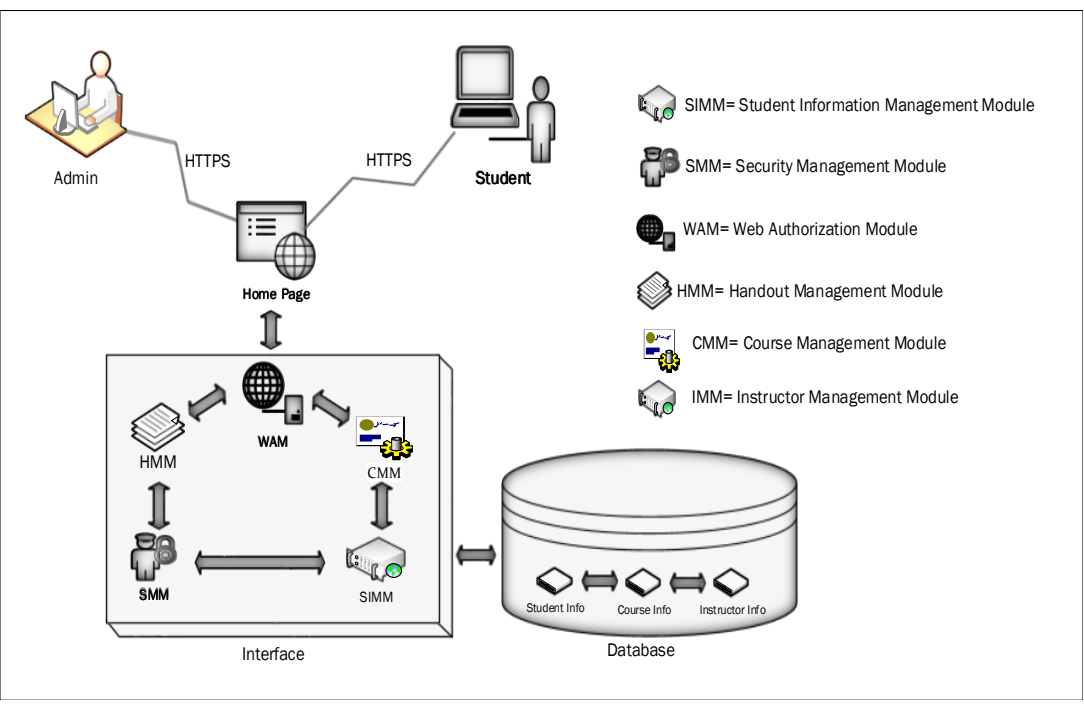

Fig. 1. Handout management System

an avenue for stakeholders to track progress of project during its development phase.

\section{SYSTEM REQUIREMENTS}

There are considered different kinds of requirements named functional and non-functional requirements. Now, those are described as follows:

(1) Functional Requirements: Handout management system aims how to upload and download different class materials (notes/files/documents) in this system. To build and run these application, there are needed some functional requirements like course contents, information of course instructors, records of student and secured protocol.

(2) Non-Functional Requirements: Non-functional requirements are divided into three categories. Those are described as follows:

-Performance Requirements: Handout management system are used to manage students and handout of instructors in the organization. Therefore, it is expected that this system would perform functionally to specify all the requirements.

- Safety Requirements: Handout management system may crash at any time due to virus or operating system failure. Therefore, it is required to take backup of this system.

-Security Requirements: Different people are viewed required information from this system. Depending on the category of user, access rights are decided. If user is an administrator then he can be able to append, modify the data in this system more efficiently. All other users (instructors and students) only have the rights to retrieve the information.

\section{METHODOLOGY}

Handout management system consists of some modules which are web authorized module(WAM), student information management module(SIMM), instructor management system(IMM), course management module(CMM), handout management module(HMM) and security management module(SMM). Different modules of handout management system are depicted in figure 1 Those modules are described briefly as follows:

-Web Authorized Module: Authorization is a process where user has permitted to use different resources in the system. In handout management system, there are considered three authorized users which are administrator, instructors and students. It is verified by user requests who are wanted to access in the system. When user are verified, he can access in the system.

- Student Information Management Module: Student information management module (SIMM) provides a simple interface to maintain student information. It provides individual accounts to the student which is needed to explore his courses which are assigned in the professional master's program. Besides, it deals with student's personal information, academic reports, assigned courses and other resources too.

-Instructor Management Module: Instructor management module (IMM) is used to maintain faculty members, trainers information who are delivered their handouts. In this case, Instructor can visualize which course is taken by him and can upload their handouts in those particular courses.

-Course Management Module: Course management module (CMM) is used to handle course curriculum, credit hours, contents that is helpful to get required information about different courses for students and instructors.

- Handout Management Module: Handout management module (HMM) is a core module of this system which manage all 
notes/slides/e-book/document in the system. All the class notes are provided by the instructors according to the date and course code of given lecture. Students can watch and download slides, e-book and documents of a course when they are assigned in these courses.

- Security Management Module: There are considered https protocol to send and receive different contents between user and server.

\section{RESULT AND DISCUSSION}

In this experiment, there are implemented secure web based handout management system on the web. This application contains some information related to courses and students of professional master's program at Department of Computer Science and Engineering in Jahangirnagar University. Then, those data are analyzed to exemplify the performance of this secure web application. There are used core i5 processor, 4 GB RAM based HP Probook 4540s laptop with Windows 10 operating system.

In figure 2, there are represented a relationship between upload time and download time about different types of files in this system. Handout of courses are uploaded by the instructors and students are downloaded those files as their requirement. There are taken different type of files and uploaded them (29 MB, 40.7 MB, 52.4 MB, 70.3 MB and 82.5 MB) sequentially in this system and taken upload times such as $0.71 \mathrm{~s}, 0.97 \mathrm{~s}, 1.41 \mathrm{~s}, 1.59 \mathrm{~s}$ and $1.89 \mathrm{~s}$ approximately by a google chrome plugin/extension named "Page Load Time". Then, we have taken download time using stopwatch such as $1.15 \mathrm{~s}, 1.55 \mathrm{~s}, 2.35 \mathrm{~s}, 2.45 \mathrm{~s}$ and $2.95 \mathrm{~s}$ approximately according to the size of files.

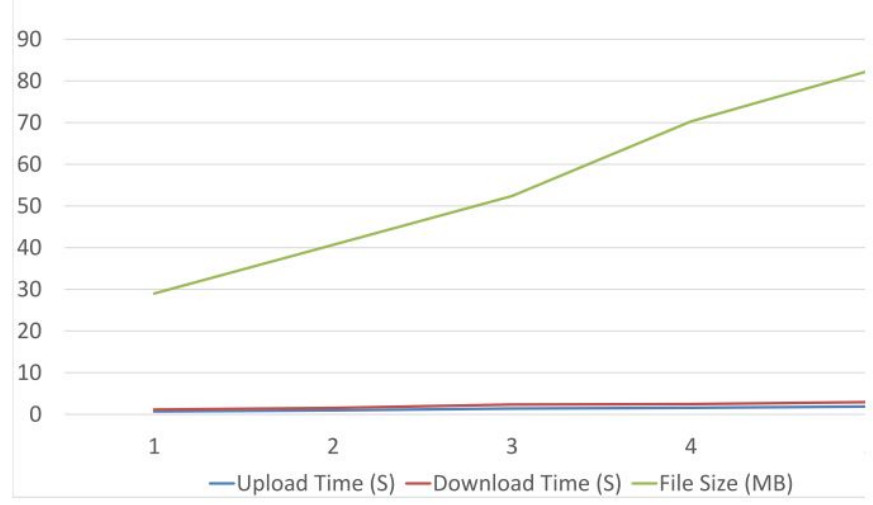

Fig. 2. relationship among upload time, download time and file size

In this experiment, different upload and download times are represented comparability of different files by passing secure https protocol in this system. It is also observed that upload time is less than its download time.

Besides, there are offered many courses but all courses are not showed same demand to the students in the professional master's programs. So, some highly demandable course with number of
Table 1. Course Description

\begin{tabular}{|l|l|l|}
\hline Course No. & Course Title & Students \\
\hline PMSCS 653 & Advance Database Management System & 35 \\
\hline PMSCS 655 & Web Design and Programming & 25 \\
\hline PMSCS 657 & Parallel and Distributed Computing & 30 \\
\hline PMSCS 658 & Mobile and Wireless Communication System & 30 \\
\hline PMSCS 663 & Digital Image Processing & 20 \\
\hline PMSCS 675 & Network Security & 40 \\
\hline PMSCS 677 & Neural Networks & 25 \\
\hline
\end{tabular}

student are showed in table 5

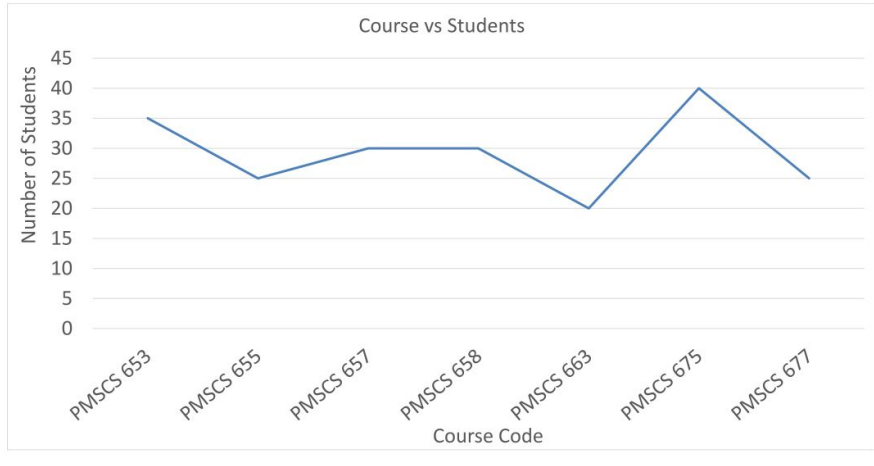

Fig. 3. relationship between courses and students

In figure 3, there are shown the relationship between courses and students who are taken different courses. It is represented a graphical overview how many students are assigned in different courses. Besides, it is also represented which course is less interested and more demandable to the students in the professional master's program at the Department of Computer Science and Engineering in Jahangirnagar University.

\section{CONCLUSION AND FUTURE WORKS}

In this work, there are represented a web based secure handout management system that provides information of course, student, instructor and calculate upload and download time different course contents on the web. Besides, interest of different courses by students are represented using the data of this system. But this system has some limitations. There are used simple https protocol but not used any high secured protocol in this application. In future, high secure protocol/algorithm may be added to this application to enter student information, upload and download course handout very easily.

\section{REFERENCES}

[1] Bimal Aklesh Kumar. Thin client web-based campus information systems for fiji national university. arXiv preprint arXiv:1102.0583, 2011.

[2] SR Bharamagoudar, RB Geeta, and SG Totad. Web based student information management system. International Journal of Advanced Research in Computer and Communication Engineering, 2(6):2342-2348, 2013. 
[3] Tasleem Nabiwale Namita Naik Suraj Patil Bahubali Akiwate, Ayazahmed Patel. Design a web based student information management system using mean stack. International Journal of Advanced Research in Computer Science and Software Engineering, 6(5), 2016.

[4] Rui Duan and Mingsheng Zhang. Design of web-based management information system for academic degree \& graduate education. Integration and Innovation Orient to E-Society Volume 2, pages 218-226, 2007.

[5] Giansalvatore Mecca, Paolo Atzeni, Alessandro Masci, Giuseppe Sindoni, and Paolo Merialdo. The araneus webbased management system. In ACM SIGMOD Record, volume 27, pages 544-546. ACM, 1998.

[6] Wei-Kuang Huang and Vijayalakshmi Atluri. Secureflow: a secure web-enabled workflow management system. In Proceedings of the fourth ACM workshop on Role-based access control, pages 83-94. ACM, 1999.

[7] Adeel Akbar Memon, Chengliang Wang, Muhammad Rashid Naeem, Muhammad Tahir, and Muhammad Aamir. A new web based student annual review information system (saris) with student success prediction. arXiv preprint arXiv:1406.4842, 2014.

[8] Prafulla S Yevale, Miss Mohini R Malpure, Ram S Varma, and Miss Kirti P Wadje. Web based college information monitoring system using java (digicampus). Imperial Journal of Interdisciplinary Research, 2(6), 2016.

[9] Aaron A Izang, Chinyere C Ihesiulo, Miracle Ofuru, and Chukwuebuka Okafor. A web-based project management system. International Journal, 6(4), 2016. 